\title{
Fuzzy Hybrid Deliberative/Reactive Paradigm (FHDRP)
}

\author{
Hengameh Sarmadi \\ Department of Electrical Engineering \\ Vienna University of Technology (TU-Wien) \\ Karlsplatz 13, A-1040 Vienna / Austria
}

\begin{abstract}
This work aims to introduce a new concept for incorporating fuzzy sets in hybrid deliberative/reactive paradigm. After a brief review on basic issues of hybrid paradigm the definition of agent-based fuzzy hybrid paradigm, which enables the agents to proceed and extract their behavior through quantitative numerical and qualitative knowledge and to impose their decision making procedure via fuzzy rule bank, is discussed. Next an example performs a more applied platform for the developed approach and finally an overview of the corresponding agents architecture enhances agents logical framework.
\end{abstract}

\section{Introduction}

The definition of the agents world could be based on their social rules, communication cooperation and negotiation between them and their pursuance to achieve the defined (pre-given) goals. The central concerns on this area refer from one side to the individual design aspects for developing the design task and leading to an improved behavior and from another side to their social rules, cooperation of the individuals, and consideration based on the relationship between individual and overall social behaviors. The design aspect should be suitable for time constraint environment and interactions with environment in order to make agents capable to reconfigure and recover from changes due to environment and satisfy other flexible design criteria. Intelligent agents acquire information from the world interface and are able to perform tasks which are supposed to meet deadline on average [2].

In heterogonous approach the agents may differ from each other to ensure multi robot coordination. Self regulation agents concept is embedded within the environment and their autonomous action meets the design objectives. Part of the problem from individuality point of view refers to path planning and navigation, which implies the complexity of the problem and represents the physical limitations of robot platform.

In a human-based model of agents there is a close dependent between the deliberation, reaction and decision making and the agents relationship functional structure models these criteria [8]. If we face the unstructured or local environment the reactive planning is the most appropriate execution meanwhile in a knowledge rich environment (global/open worid) a hierarchical paradigm works better based on deliberation process of global information and agent specific abstraction. Deliberation functions could not extend independently of reactive behavior and vice versa. Hybrid architectures benefit both concepts of reactive and deliberative paradigm [5]. 


\section{Incorporation of Fuzziness into Hybrid Paradigm}

As stated above the agents should have some reactive design-base due to changes in environment, which in turn may effect and result in some limitation in the agents (local) goals and deliberation paradigm, which through reasoning procedure and intention tend to lead to action to achieve the goals (mean-ends). Therefore we need to balance between goal directed (deliberative) and reactive paradigm. Fuzzy approach can perform a suitable area for considering both these aspects, through which our decision function is a fuzzy one which proceeds the action design choice influenced by history and reconsideration and makes the agents enable to develop cognitive functions for evolution of intelligence.

Deliberation and reactivity face with problems of multiple conflicting criteria and multiple objectives. With incorporation of fuzziness into hybrid paradigm we can make decisions with vague, uncertain and inexact objects and extract the human knowledge in planning architecture without articulating an application-based world model and prepare a determinative interpretation from probability and randomness. Fuzzy approach profits knowledge representation about how the agents represent their world, plan and solve problems in close/open world and is appropriate to experiment on bold (never reconsider) to cautious (constantly reconsider) agents, since the decision procedure attempts to degree.

\subsection{Development of the Concept of FHDRP}

The decision making strategy will be based on a deliberation-reaction fuzzy rule bank with strategy acquisition in extracting the fuzzy rules incorporating with real time reasoning $[3,4,6]$ (we can learn the fuzzy rules from experiences with numerical and/or linguistic sample data with enhancing the system profiting the ability of learning-base systems such as neural networks.).

The fuzzy deliberative/reactive rule bank could be defined as follow:

- FRB : $\quad\left(X_{i}, C_{i}, A_{i}\right), \quad i=1,2, \ldots, n$.

Where:

-Deliberation state conditions: $X_{i}=\left\{X_{i 1}, X_{i 2}, \ldots, X_{i m}\right\}$.

With deliberation fuzzy set: $D=\left\{\left(X, m_{D}(X)\right) \mid X \in \Omega\right\}, \Omega$ is the universe of the deliberations.

-Reaction state conditions: $C_{i}=\left\{C_{i 1}, C_{i 2}, \ldots, C_{i s}\right\}$.

With reaction fuzzy set: $R=\left\{\left(C, m_{R}(C)\right) \mid C \in \Psi\right\}, \Psi$ is the universe of the reactions.

-Action through deliberation and reaction: $A_{i}=\left\{A_{i 1}, A_{i 2}, \ldots, A_{i k}\right\}$.

With action fuzzy set: $A_{C}=\left\{\left(A, m_{A_{C}}(A)\right) A \in \Gamma\right\}, \Gamma$ is the universe of the actions.

The fuzzy hybrid paradigm can be defined as the projection:

$D \times R \rightarrow A_{C}$. Where $\mathrm{D}$ is deliberation, $\mathrm{R}$ is reaction and $A_{C}$ is action power set.

The i-th fuzzy rule seems like: 
If ( $X_{i 1}$ is $f_{x 1}$ and $X_{i 2}$ is $f_{x 2} \ldots$ and $X_{i n}$ is $\left.f_{x n}\right)$ and if $\left(C_{i 1}\right.$ is $f_{c 1}$ and $C_{i 2}$ is $f_{c 2} \ldots$ and $C_{i s}$ is $f_{c s}$ ) then $A_{i}$ is $f_{a i}$.

So the behavior could be written as: $B e h \subseteq R \times R \times R$, ordering on $\mathrm{R}$ and could be defined as:

- Béh $=\left\{\left(X_{i}, C_{i}, A_{i}\right) \mid X \in D, C \in R, A \in A_{C}\right\}$, or

$-B e h=R \bigcup_{i=1}^{P} X_{i 1} \times X_{i 2} \times \ldots \times X_{i n} \times C_{i 1} \times C_{i 2} \times \ldots \times C_{i s} \times A_{i 1} \times A_{i 2} \times \ldots \times A_{i k}$.

The parallel associative inference will fire each fuzzy rule in parallel but.to different degrees.

As a traditional defuzzifier approach the max-height method can be used:

$$
-m_{A_{C}}\left(A_{\max }\right)=\max _{i=1}^{p} m_{A_{C}}\left(A_{i}\right) \text {. }
$$

The defuzzification procedure can be completed with the priority rule or the subsumption theory developed by Brooks [1]. Therefore we get the inhibition relation in the hierarchy as follow:

$A_{i} \prec A_{j}$ if $\left(A_{i}, A_{j}\right) \in \prec$, and we read it:

$A_{i}$ inhibits $A_{j}$ or $A_{i}$ is lower in hierarchie than $A_{j}$.

\subsection{Example}

As an example (modified from [7] and [8]):

Suppose the objective is to collect samples in an indoor environment of a particular type in a predefined place. The location of the samples is not known. A number of autonomous swarm agents are for this problem available which can go around and collect the samples. Furthermore the terrain is full of obstacles. This organized team of robots can in turn negotiate and cooperate together and divide up the task collaborating with a common coordinator and the individuals have autonomous decisions and navigations.

In this problem we face with a mix of path and deliberation/reaction planning and we need a path planning algorithm (for further detail see next section) as well, which sufficiently represents the terrain.

We could extract some fuzzy deliberation/reaction rules considering agents specific criteria and agents cooperation, for example:

-Deiiberation (if there are more samples in one direction, Move-to-that-direction) and Reaction (if near a sample and obstacles or other agents are far, Speed-up-towardsthe-sample).

-Deliberation (if the obstacle is far, Choose-the-up-gradient-direction-toward-thesample, Move-to-sample) and Reaction (if the obstacle is very close, Change-thedirection).

-Deliberation (if your partner is closer than you to a sample and there are fewer obstacles in his way, Communicate-with-your-partner and Let-him-to-pick-it-up). 
-Deliberation (if another agent near you has more frustration, Go-in-his-direction and Pick-up-the-sample).

-Deliberation (if many other agents in one direction, Choose-another-direction) and Social rule (if another agent near you Wait or Turn-to-the-left).

And so on.

We can interpret for all of the linguistic notions mentioned above, which can not be exactly described, such as: more, near, far, close, few,... the corresponding fuzzy sets and with defining the degree of indeterminacy articulate numerical data structure for partial occurrence of events or relations and have a quantitative interpretation from probability and randomness.

Finally pure reactive rules such as: Avoid-obstacle, finding a sample Take-it and carrying samples and at the base Drops-the-samples have the most priority and complete the decision procedure with inhibition characteristics.

\section{FHDRP Control Architecture of the Agents}

The design-base is appropriate for real time execution and the state hierarchy develops a layered intelligent structure, whereby each layer could be interpreted as software agent or function in order to develop logic based concepts of robots and assure a modular construction for replaning and adapting the configuration.

The control strategy suggested and the proactive behavior could be based on supervisory control on agent level. Coordinated decision must be suitable for application specific behavior on user level program embedded in the controller at run time. Application specific information, control algorithm and planning strategy specify the agent code. We should define the optimal in favor of our data base, rule base and changes that will occur in the environment.

The approach (fig. 1) is based on SENSE then PLAN ACT, whereby the sensed information goes through planning layer and by means of directives translates to actuator commands on a hierarchical paradigm. One of the inputs to the systems will be sequences of environment states or percepts through which the control rules will extract some deliberative/reactive behaviors. Action rules translate to the effectors via corresponding sensor system through pattern of motor schema action. The developed concept is applicable to both local and cooperative planning.

The fuzzy approach develops the social ability and satisfies the abilities due to uncertainty in the world model and provide a balance between goal directing and reactivity and interactions between the agents in order to coordinate and control them, using Quantitative numerical and qualitative knowledge. Fuzzy rules consider attention, reasoning, and information collection. We need real time processor to proceed with fuzzy reasoning about the global state to select the best behavior.

The behavioral manager plans which behavior to use in order to progress to the goal and there are assumptions mappings from global data structure (sensory inputs) to behavior generation.

Behaviors are inherently parallel and distributed and the goal directed approach is a sequence of generic behavior and updating the behavior.

Sequencer as in the general hybrid architecture generates the set of behaviors, adapts it with managerial style and subdivides the deliberation based on the control scope and enables the system to develop a behavior based control for coordinating 
planful activities with real time behavior for dynamic positioning, navigation (behavior based opportunity to change direction of navigation) and considering the goals, resources and timing constraints.

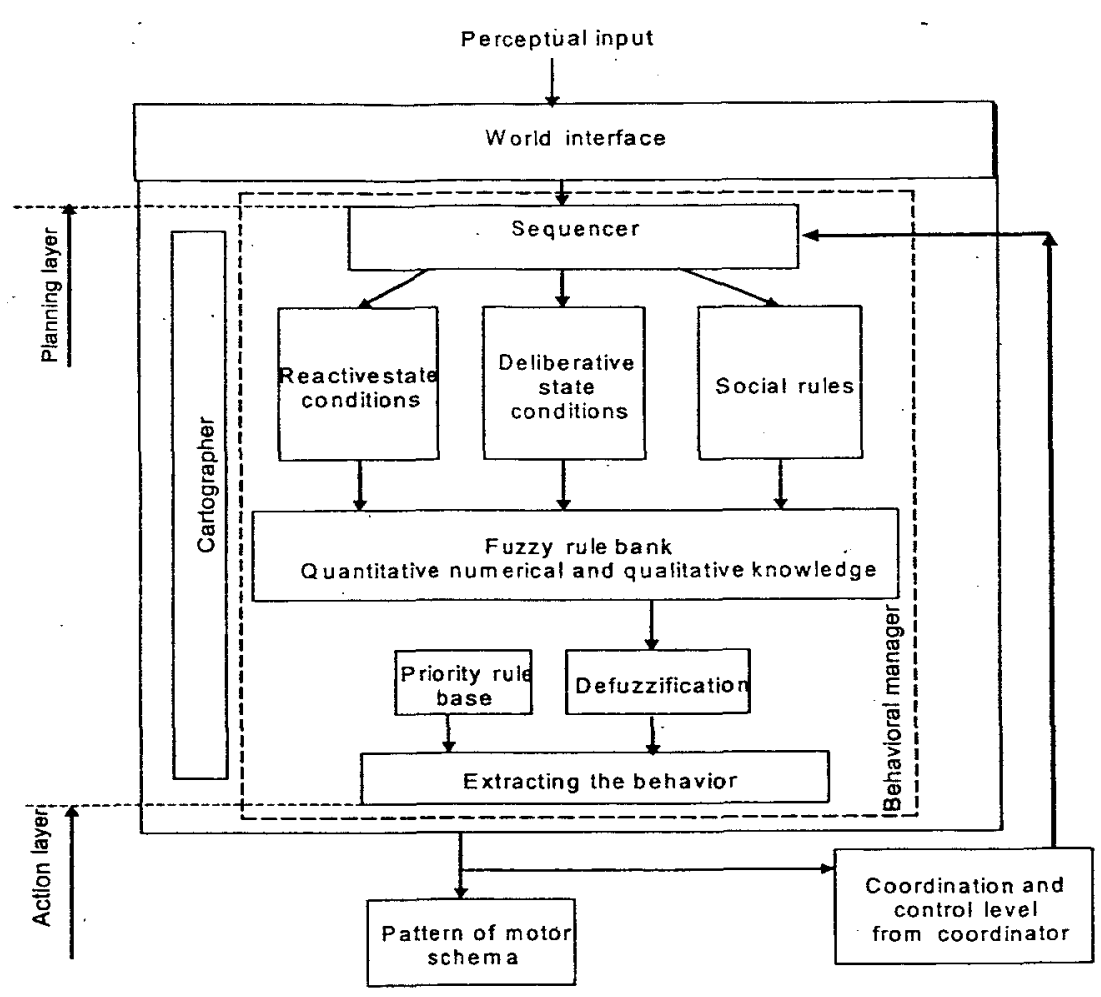

Fig. 1 FHDR Control architecture of agents

Cartographer is responsible for information collection (data structure) and path planning. Physical location of agents and the coordinated control program will be part of global knowledge and shared data structure. To find the optimal path in the configuration space, event noticeable by reactive system would trigger event-driven repianing.

Path generation algorithm will generate a pre completed path with a hill climbing algorithm to reach to the target position, where the target position is given by the strategy system and behavioral controlling of the moving direction of the agent is based on the direction of the target point and agent actual coordinates [6].

If an obstacle blocks the path, the path is replaned and optimized by computing the optimal route and decomposing it into waypoints [5], a goal to reach. After 
reaching each waypoint next goal is computed and cartographer gives the sequencer a set of waypoints to make a qualitative navigation possible.

Solutions to the problems such as interference, member unproductive or failed, agents interactions and communications, individuality and autonomy, emergent behavior and heterogeneity could be in collaborating with the coordinator. Coordinator defines new goals and sets the strategical plans, which lead to tactical instructions and coordinate the relationship between strategy and agents set of tactical behavior through social rules. Coordinator can modify the relationship between the behaviors of agents: one strategy and several tactics.

\section{Future Directions}

For a later work we could enhance the agents architecture with learning-base distributed AI systems such as neural networks to learn from experiences and new data and be able to improve the agents behavior. The leaming system should develop the ability of on-line learning in time critical environment using qualitative abstraction, symbolic learning algorithm and rules generation and challenge the autonomous learning of sequential behavior and gain some skills to carry out the plan for more robustness and performance monitoring. We could also investigate on stability, redundancy and complementary of the system.

\section{References}

1. R. A. Brooks. A Robust Layered Control System for a Mobile Robot. IEEE Journal on Robotics and Automation, 2(1) (1986) 14-23

2. R. A. Brooks. Intelligence without Reason. Proceeding of the Twelfth International Joint Conference on Artificial Intelligence (IJCAI-91), Sydney, Australia (1991) 569 595

3. H. Hellendoorn. Reasoning with Fuzzy Logic. University of Technology, Delft (1992)

4. E.H. Mamdani. Application of Fuzzy Logic to Approximate Reasoning using Linguistic Synthesis. IEEE Transaction on Computers C-26, no.12 (1977) 1182-1191

5. R. R. Murphy. Introduction to AI Robotics. Cambridge, Mass.: MIT Press (2000)

6. H. Sarmadi. An Approach to a Supervised Neural Network-based Fuzzy Controller. PhD Dissertation, Vienna University of Technology, Vienna (1995)

7. L. Steels. Cooperation between Distributed Agents through Self Organization, Decentralized AI. Proceeding of the First European Workshop on Modeling Autonomous Agents in a Multi-Agent World, Amesterdam, the Netherlands (1990) $175-196$

8. M. Wooldridge. Intelligent Agents: the Key Concepts. Multi-Agent Systems and Application II, $9^{\text {th }}$ ECCA-ACAI/EASSS 2001, Prague, Czech Republic (2002) 3-43 